\title{
LA CONTRIBUCIÓN DE LA SOCIEDAD ASTRONÓMICA DE BARCELONA EN LA DIFUSIÓN DE LAS OBSERVACIONES METEOROLÓGICAS EN CATALUNYA (1910-1923)
}

\author{
Marc J. Prohom Duran \\ Área de Climatología \\ Servei Meteorològic de Catalunya
}

\section{RESUMEN}

La Sociedad Astronómica de Barcelona (1910-1923) se fundó con el objetivo de desarrollar y difundir el conocimiento científico en la ciudad de Barcelona y, por extensión, Catalunya. A pesar del peso mayoritario de los estudios astronómicos durante los primeros años, la observación meteorológica pasó poco a poco a ser una de sus actividades más destacadas, dejando un legado de gran interés climático. Gracias al impulso de sus socios y colaboradores, se amplió de manera espectacular el número de estaciones meteorológicas (buena parte de ellas pluviométricas) llegándose a los 224 observatorios en 1918, se uniformizaron métodos y normas de observación e instalación de instrumentos, y se popularizó la meteorología entre la población. En este artículo, y mediante el estudio detallado del centenar de boletines que publicó esta institución durante su corta pero intensa vida, se analiza el papel determinante que jugó en la instauración de la Red Pluviométrica Catalana, sin duda de las de mayor densidad de España para la época y que ha dejado su huella en el presente.

Palabras clave: Observación meteorológica, red pluviométrica, Catalunya, primer cuarto de siglo XX.

\begin{abstract}
The Astronomical Society of Barcelona (1910-1923) was founded with the main objective of developing and spreading the scientific knowledge in Barcelona and Catalonia. During the first years, the astronomical studies were the most common, but little by little the meteorological observation became the priority, leaving knowledge of great climatic interest for the following researchers. Thanks to the impulse of their members and contributors, the Catalan meteorological network increased to the number of 224 observatories in 1918, methods of observation and rules for weather instrument installation were suggested, and the meteorology became popular amongst the population. In this paper, the role played by this Society in the establishment and extension of the
\end{abstract}


Catalan Rainfall Network is analysed through the detailed study of one hundred bulletins published. Certainly, it was the densest network in Spain during the first decades of the $20^{\text {th }}$ century, and has left its mark on the present.

Key words: Meteorological observation, rainfall network, Catalonia, first quarter of $20^{\text {th }}$ century.

\section{Los precedentes}

Catalunya ha sido siempre un territorio muy sensible al estudio de la meteorología local, tanto a capítulo particular como colectivo. Así, desde la segunda mitad del siglo XVIII hay constancia del interés por la observación meteorológica de la mano de médicos como Francesc Salvà, Pere Vieta o J.R. Campaner, eruditos ilustrados como Martí i Franquès, o matemáticos como Llorenç Presas (1811-1875) quienes realizaban precisas observaciones en la ciudad de Barcelona y otras localidades catalanas (Barriendos, 2001; Puig, 1995). Este hecho ha permitido disponer de la serie instrumental más prolongada de España (Barriendos et al., 1997; Rodríguez et al., 2001). A pesar de estas loables y valiosas contribuciones, no es hasta la segunda mitad del siglo XIX cuando aparecen las primeras e incipientes redes de observación. Así, en España se publica en 1858 el primer «Anuario Estadístico de España», en el que se recogían resúmenes meteorológicos de 11 estaciones peninsulares: Madrid (Real Observatorio), Instituto de Alicante y Universidades de Barcelona, de Granada, de Oviedo, de Salamanca, de Santiago, de Sevilla, de Valencia, de Valladolid y de Zaragoza. Posteriormente, el Observatorio Astronómico de Madrid publicó a partir de 1866 el «Resumen de las Observaciones Meteorológicas» pasando de 22 estaciones, en 1867, a 64, en 1885 (García de Pedraza y Giménez de la Cuadra, 1985). En 1887, el nuevo Instituto Central Meteorológico debía asumir la gestión y mantenimiento de la citada red, pero diversos problemas presupuestarios no lo hicieron posible hasta veinte años después (1904), cuando el Observatorio y el Instituto pasaron a depender del Instituto Geográfico y Estadístico.

En Catalunya, además de las observaciones que se realizaban en la Universidad de Barcelona (desde 1861), en el último tercio del siglo XIX hay constancia de otros puntos de observación, aunque sin llegar a conformar una red de observatorios. Entre ellos cabe destacar las observaciones que se realizaron en la Casa Misión de Balaguer, desde 1862 o en Vilert (comarca del Pla del Estany) desde 1867 (Febrer, 1930). A pesar de ello, el primer paso firme para la creación de una red catalana de observatorios no se realiza hasta finales de 1893, cuando el director de la Granja Experimental de la Diputación de Barcelona, Hermenegildo Gorría, expone la necesidad de aumentar el número de estaciones meteorológicas para dar un mejor servicio a la agricultura (Sureda, 2003). Así fue como en 1895 se creó la «Red Meteorológica de Cataluña y Baleares» con un total de 14 observatorios, publicando sus resúmenes en la revista del Instituto Agrícola Catalán de San Isidro. Con la colaboración del Dr. Eduard Fontserè y de la Diputación Provincial de Barcelona, la red fue creciendo hasta unos cincuenta observatorios repartidos por los dos territorios, siendo sus observaciones publicadas en forma de boletín (Roca et al., 2004).

Hacia 1899 existió la voluntad de realizar un paso más allá mediante la creación de una «Sociedad Meteorológica del N.E. de España». Incluso se redactaron los estatutos, pero la falta de subvención por parte de la Diputación frenó en seco esta iniciativa y no se llegó a publicar ni un solo boletín. A pesar de ello, Rafael Patxot, conocido mecenas de las ciencias catalán y asimismo observador del «Observatori Català de Sant Feliu de Guíxols», recogió parcialmente la idea y coordinó la «Red Pluviométrica Catalana», reduciendo las observa- 
ciones a la pluviometría. Esta red estaba constituida por observatorios catalanes públicos y privados, algunos de los cuales formaban ya parte de la red de la Granja Experimental. Fruto de estos esfuerzos se pudo publicar en 1908 la obra «Pluviometria Catalana», un primer testimonio de calidad sobre la climatología de la precipitación en Catalunya, con datos mensuales para el período 1896-1905 (Patxot, 1908). Unos años más tarde, esta publicación tuvo continuidad con la aparición de los resultados para el quinquenio 19061910 (Patxot, 1912).

Llegamos así al año 1910 con una red de unos setenta observatorios en Catalunya (más ocho repartidos entre Baleares, Andorra y el sur de Francia). Ese mismo año se crea la Sociedad Astronómica de Barcelona, institución que, tal y como citan Roca et al. (2004), va a suponer un hito en el asociacionismo científico catalán y que, como veremos a través del estudio del centenar de boletines que se publicaron, supondrá un salto en la difusión y expansión de la observación meteorológica en Catalunya.

\section{Los primeros pasos de la Sociedad Astronómica de Barcelona (1910)}

Constituida oficialmente el 5 de febrero de 1910, la Sociedad Astronómica de Barcelona (SAB) recogía en sus Estatutos y Reglamento, que se fundaba con la finalidad de «reunir aquellas personas que se ocupan práctica y teóricamente en Astronomía, Meteorología, Física del globo y Ciencias afines, o que se interesan por el desarrollo de estas ciencias para el bien de la cultura en general.» (Boletín de la Sociedad Astronómica de Barcelona, n. 1. BSAB-1, 1910). Se manifestaba pues la vocación divulgadora de la Sociedad en todo aquello relacionado con las «ciencias del cielo», tal y como así mismo consta en el primer boletín publicado en Julio de 1910. Esta función educadora del gran público será a lo largo de su historia una de sus principales preocupaciones. Como muestra son las numerosas conferencias y cursos que miembros de la SAB realizarán en escuelas, universidades e instituciones diversas, no sólo de Barcelona sino de diversos puntos de Catalunya (Solsona, Vic, Badalona,...), siendo algunas de ellas publicadas en el mismo boletín.

La creación de la $\mathrm{SAB}$ supuso un auténtico impacto en el mundo científico catalán del momento. Como muestra del empuje que la institución experimentó en sus inicios indicar que a finales de 1910, constaba ya de 230 socios (un 30\% de los cuales residía fuera de Barcelona). Durante los primeros años, y bajo la presidencia de Esteve Terrades, los artículos que se publicaron en los boletines fueron mayoritariamente de temática astronómica, en especial sobre astronomía práctica y observación de diferentes efemérides, como el paso del cometa Halley el año 1910, o el eclipse total de sol del 17 de abril de 1912 (Jardí, 1912). Con la llegada a la presidencia del Dr. Eduard Fontserè, considerado el padre de la meteorología catalana, el cariz y objetivos de la Sociedad sufrió una progresiva deriva hacia los estudios meteorológicos y climáticos, tendencia que mantendrá hasta su disolución.

\section{La meteorología toma protagonismo (1911)}

La presidencia de Eduard Fontserè en el año 1911 (y hasta 1913) coincidió con la aparición de algunas tímidas aportaciones de carácter meteorológico. De hecho, la primera noticia en este sentido aparece en el boletín número 8, de marzo de 1911, cuando se anuncia la recepción diaria en la sede de la $\mathrm{SAB}$ de los datos meteorológicos observados durante la mañana en Europa, Azores y norte de África, datos que eran cedidos por el Observatorio Central Meteorológico (OCM) de Madrid. Igualmente, en el posterior número de abril del 
mismo año figura el primer artículo sobre temática exclusivamente meteorológica: «Las exploraciones atmosféricas en España» a cargo del capitán de ingenieros Emilio Herrera.

El verdadero giro de la Sociedad hacia el fomento de los estudios en meteorología y de la difusión de la red de observadores, se pone de manifiesto en un artículo de Fontserè en mayo de 1911. Allí anuncia el papel vital que la SAB jugará en la creación de una red pluviométrica a nivel estatal, fomentada por el OCM de Madrid y el Instituto Geográfico. En este sentido, escribe: «... creo que nuestra Sociedad ha de poner todo su empeño en que el número de observadores de la nueva Red española sea muy crecido, recomendando a sus socios de toda España la cooperación personal, y ayudando a la empresa por medio de su Comité de vulgarización.» (Fontserè, 1911). Según Roca et al. (2004), este artículo habría sido escrito por José Galbis, amigo personal de Fontserè y jefe del OCM, pero firmado por Fontserè para no crear diferencias con otros colectivos científicos. Así, en la reunión mensual de 9 de abril de 1911, la Junta Directiva acuerda considerar de interés preferente fomentar las iniciativas del OCM y las de su director, recomendando a sus socios tomar parte en esta empresa, conjuntamente con el Observatori Català de Sant Feliu de Guíxols, a cargo de Rafael Patxot.

En los meses posteriores a este primer paso, la SAB se puso en contacto con la British Rainfal Organization, solicitando información relativa al funcionamiento de esta organización para adaptarla a las necesidades de la recién creada Red Pluviométrica Española (BSAB, n. 12). Paralelamente al impulso de la red de observadores, la vocación divulgadora de la meteorología tiene un nuevo episodio cuando a partir de Junio de 1911 empieza a exponerse en el Ayuntamiento de Barcelona un mapa meteorológico diario de Europa, elaborado por los socios Álvarez-Castrillón y Rubio. La primera aparición de datos meteorológicos se constata en el boletín número 13, donde en la sección «Noticias» se informa de las observaciones pluviométricas en Begur (Costa Brava), primera estación creada directamente por la SAB y a cargo del socio Francesc Pi. En este breve escrito constan las precipitaciones diarias caídas entre agosto y octubre de 1911, destacando la gran «parquedad de las precipitaciones acuosas» de la primavera y verano del citado año. A partir de esa fecha y durante toda la década, aparecerán publicados regularmente los totales pluviométricos mensuales de diferentes observatorios, la mayor parte impulsados directamente por la SAB, así como otras informaciones meteorológicas, tales como episodios de tiempo severo (temporales de viento, nevadas y granizadas) u observaciones fenológicas.

\section{Consolidación y ampliación de la red pluviométrica catalana (1912-1918)}

La constatación que los estudios propiamente meteorológicos iban tomando cada vez más protagonismo, la tenemos con la creación, en enero de 1912, de la «Comisión especial de Meteorología exógena y endógena», dirigida por el Dr. Ramon Jardí y que funcionará como órgano autónomo de la SAB. Entre sus principales cometidos destaca la de organizar y gestionar la ampliación de la red pluviométrica, así como de centralizar la ingestión de los datos que ésta generaba. Así, Salvador Rauric, secretario y entusiasta impulsor de la Sociedad, realiza a principios de ese año, un viaje a la comarca del Baix Empordà (Girona) con el objetivo de crear cinco nuevos observatorios pluviométricos en Palamós, Palafrugell, la Bisbal, Pals y Torroella de Montgrí, a parte del de Begur, creado ya el año anterior (BSAB18, 1912). Durante ese viaje, Rauric gestiona la cesión a la SAB del material astronómico del Observatori Català de Patxot, y realiza los primeros pasos para el traspaso, año y medio más tarde, de la gestión de la red que Patxot coordina. Meses después, el instrumental meteorológico del citado observatorio fue cedido a los padres benedictinos de la Abadía de Montserrat quienes instalaron en verano de 1913 el anemómetro Bourdon-Patxot en la cima 
del pico de Sant Dimes (a 920 m de altura), en la sierra de Montserrat. Ese mismo año, la citada comisión se pone en contacto con el profesor de la Universidad de Montpellier, Dr. Moye, experto en el conocimiento de la climatología de las regiones pirenaicas.

Un ejemplo palpable de las inquietudes que mostraba buena parte de la sociedad catalana de la época por el conocimiento del clima del país, y a las que la SAB daba contada respuesta, la encontramos con la creación de una nueva estación meteorológica en Sant Hilari Sacalm (comarca de la Selva, Girona). En otoño de 1912 y gracias a la contribución popular de los vecinos y veraneantes de la villa, se creó la estación con una dotación instrumental completísima y que procedía en buena medida de la Exposición de Estudios Lunares realizada por la SAB entre mayo y junio del mismo año, en el Paraninfo de la Universidad de Barcelona (BSAB-23, 1912).

A lo largo de sus años de existencia, la SAB tuvo estrechos lazos de colaboración con la «Real Academia de Ciencias y Artes de Barcelona» (RACAB) siendo algunos de sus socios, miembros también de la citada academia. Así, en el boletín de octubre de 1912 se anuncia la iniciativa de la RACAB de organizar, en las provincias catalanas, una red de estaciones para los estudios de la meteorología agrícola. Fruto de las conversaciones con el jefe del Servicio Nacional de Meteorología (antiguo OCM), Sr. Galbis, se acuerda que estas estaciones realicen conjuntamente con las observaciones pluviométricas y termométricas ordinarias, observaciones de insolación mediante el uso de heliógrafos Campbell y de las temperaturas del subsuelo, desde la superficie hasta la profundidad de un metro y medio. La estación meteorológica agrícola de Riudabella, en la localidad tarraconense de Vimbodí, y que funcionaba ya desde 1908, es un buen exponente del éxito de esta iniciativa.

Además de la preocupación por la creación de nuevos puntos de observación, la SAB impulsa, a través de sus asociados, la recuperación de observaciones meteorológicas antiguas. Así es como el observador de Agullana (Alt Empordà, Girona), L. Marià Vidal, recupera datos medidos en esta población desde septiembre de 1906 y que restaban inéditos hasta esa fecha (BSAB-24, 1912). También el socio B. Almirall informa de la existencia de registros pluviométricos mensuales en la población de Martorell, desde 1899 a 1913 y que son publicados en el boletín de la Sociedad (BSAB-41, 1914).

En la reunión general del día 26 de julio de 1913, se informa de lo siguiente: «en virtud de un acuerdo entre D. Rafael Patxot y la Sociedad Astronómica de Barcelona, esta última acepta a su cargo la honrosa misión de continuar recogiendo anualmente los datos pluviométricos de la Red que el Sr. Patxot viene fomentando en Cataluña desde el año 1896, trabajos que, como es sabido, sirvieron a su autor para la publicación de sus notables obras sobre «Meteorología Catalana». La «Red Pluviométrica Patxot» consta de 64 estaciones distribuidas sobre Cataluña, la cuenca Mediterránea Balear y la vertiente del Rosellón...». Con este compromiso, la SAB recoge oficialmente el testimonio de la Red Patxot y se convierte en la única institución de referencia en meteorología de Catalunya.

A partir de entonces la principal preocupación es, en la medida de lo posible, ampliar la red y uniformizarla a lo largo del territorio. De este modo, se centra la atención en las comarcas de Lleida y de los Pirineos, con una densidad de observatorios muy baja, fomentándose la distribución gratuita de pluviómetros modelo Hellmann (BSAB-33, 1913). Fruto de este esfuerzo, a finales de 1913 se crean cinco nuevas estaciones pluviométricas en Lleida, les Borges Blanques, Vallfogona de Riucorb, Prats i Sampsor y Ripoll. En esta empresa, la SAB, consciente de sus limitaciones presupuestarias, recibe el apoyo de otras entidades como son Catalana de Gas y Electricidad, Sociedad de Riegos y Fuerza del Ebro (SRFE) y Servicio Hidroeléctrico, todas ellas interesadas en el conocimiento del régimen hidrológico de los ríos pirenaicos para la explotación de sus recursos como fuente hidroeléctrica. Con la primera de las sociedades citadas, se acuerda la creación 
de cinco nuevos puntos de medida en los valles superiores de la Noguera Ribagorzana y la Noguera Pallaresa, mientras que con la SRFE se impulsa la creación, a principios de 1914, de dieciocho nuevas estaciones de alta montaña: Andorra la Vella (cuenca del Valira), Tavascan, Esterri d'Àneu, Noris, Tírvia, Espui, Sort, Sarroca, Pobleta, Tremp, la Pobla de Segur y Talarn (cuencas del Flamicell y Noguera Pallaresa), Alàs, Ponts, Lleida y Aitona (cuenca del Segre), Pont de Montanyana (cuenca de la Noguera Ribagorzana) y Faió (cuenca del Ebro). Como complemento a este cometido, el 11 de octubre de 1913 la «Comisión especial de meteorología exógena y endógena» acuerda la elaboración del mapa general pluviométrico de Catalunya en el que se localizan todas las estaciones de las que se tiene constancia, gestionadas por diferentes instituciones. No será hasta el boletín

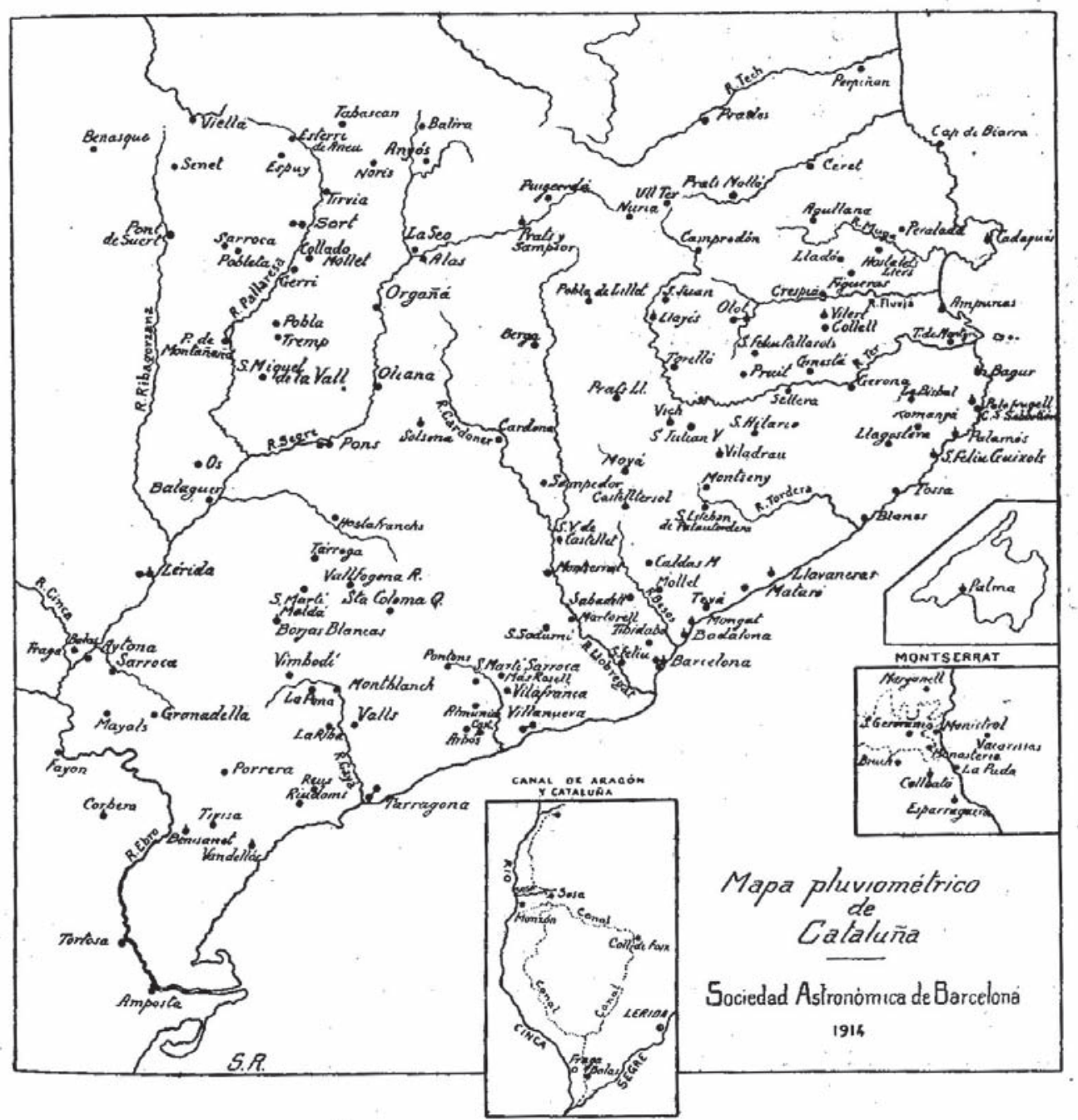

FIGURA 1. Distribución geográfica de les estaciones pluviométricas gestionadas total o parcialmente por la SAB a finales de 1914. (Mapa publicado en el boletín número 46 y elaborado por Salvador Raurich). 
de enero de 1915 cuando se publique el tan esperado mapa, y que se ilustra en la figura 1. Salvador Rauric, encargado de su elaboración apunta: «... el área del Principado exige, por término medio, la existencia de 300 estaciones, estando éstas distanciadas entre sí de 8 a 10 kilómetros, cifra esta última que puede considerarse racional para una observación eficiente del régimen de lluvias por cuencas hidrográficas y regiones orográficas, dada la complejidad de esta región española.» (BSOB-46, 1915).
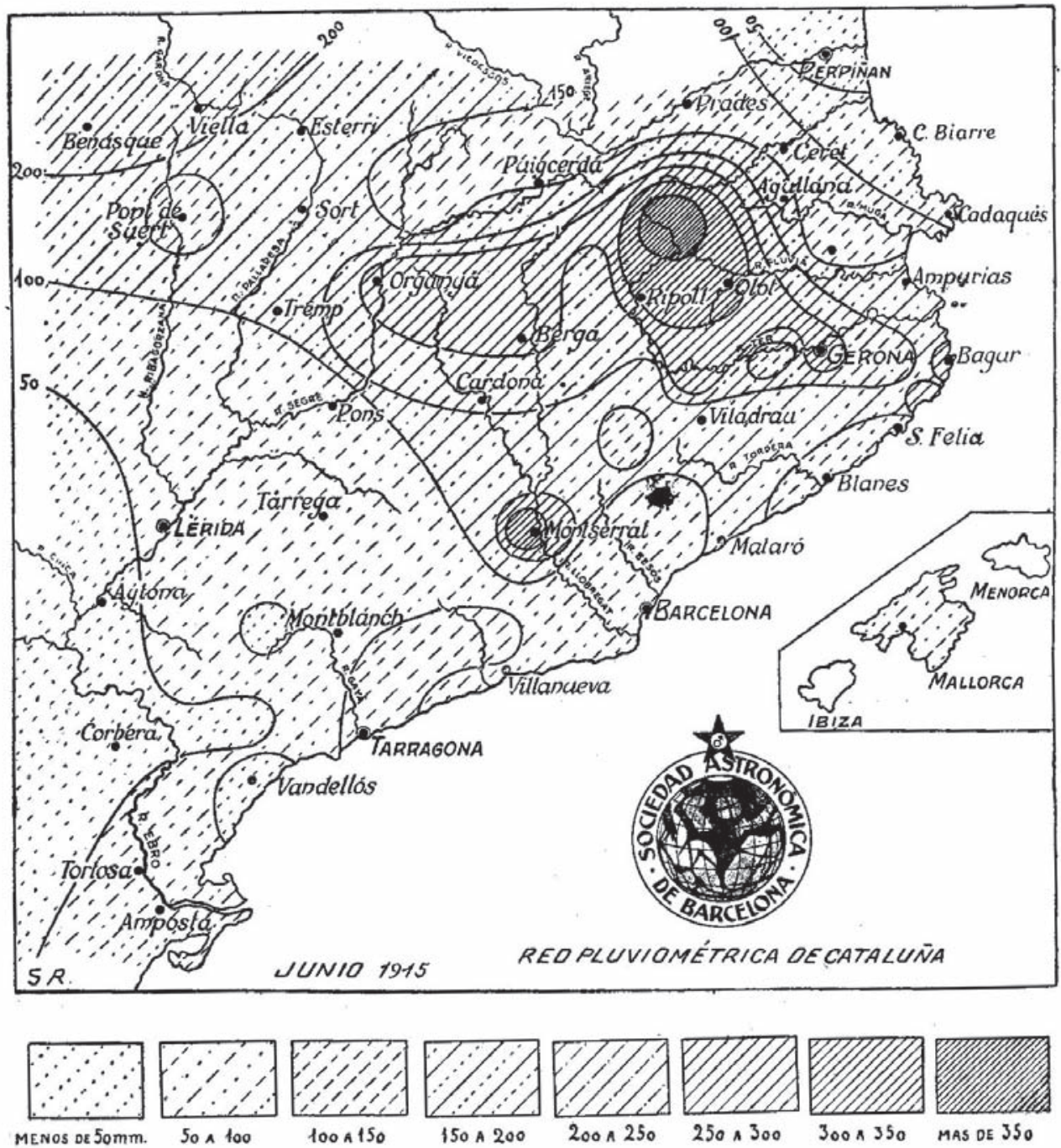

MAPa PLUVIográfico de JUNio DB 1915.

Construido con arreglo a los datos suministrados por los Señores Observadores de la Red pluviométrica de Cataluña.

FIGURA 2. Reproducción del primer mapa pluviométrico aparecido en un boletín de la SAB (octubre, 1915), correspondiente a las extraordinarias lluvias de junio de 1915. 
Unos meses después, y con motivo de las abundantes lluvias de junio de 1915, se publica el primer mapa pluviométrico (o pluviográfico) que aparece en un boletín de la Sociedad (figura 2). La ampliación progresiva de la red y una cobertura temporal nada despreciable (algunos observatorios disponían ya de cerca de veinte años de datos para esa época) hace posible el estudio detallado de ese episodio, sin precedentes para el período 1896-1914. A pesar de los escasos medios del momento, Salvador Rauric realiza en este estudio un verdadero ejercicio de reanálisis del episodio: recoge y analiza los datos de los observatorios, los compara con su promedio climático, analiza su distribución territorial y temporal y las asocia con la situación sinóptica imperante. Fruto del detallado trabajo, detecta también que algunos observatorios presentan una mala ubicación de los pluviómetros y por ello registran datos erróneos. Por ese motivo en una noticia aparecida en el boletín de enero de 1916, se acuerda remitir a todas las estaciones pluviométricas un cuestionario relativo a la instalación de los instrumentos, para así conocer con exactitud la situación de los puntos de observación, subsanar deficiencias e introducir las modificaciones necesarias para que los datos que se obtienen tengan la mayor confianza posible (BSAB-56, 1916). Asimismo, en los boletines van apareciendo instrucciones para la correcta observación de las nubes y los vientos, sobre todo aquellos que acompañan a las precipitaciones, haciendo hincapié en no sólo la importancia del número de observaciones, sino también en el máximo rigor de las mismas (BSAB-59, 1916).

A lo largo de 1915 y 1916, la SAB continúa su afán por ampliar y extender de manera uniforme la red de observadores. Así, junto con el Instituto Agrícola Catalán de San Isidro (IACSI) se distribuyen dos nuevos pluviómetros en las localidades de Vinebre (cuenca del Ebro) y de Argentona (en la comarca barcelonesa del Maresme) (BSAB-59, 1916). Igualmente, con la Cámara Industrial de Barcelona (CIB) se resuelve la creación de veinte nuevas estaciones pluviométricas en las cuencas de los ríos Ter, Fluvià y Llobregat. Este acuerdo se plasma en abril de 1916 con la creación de las siguientes
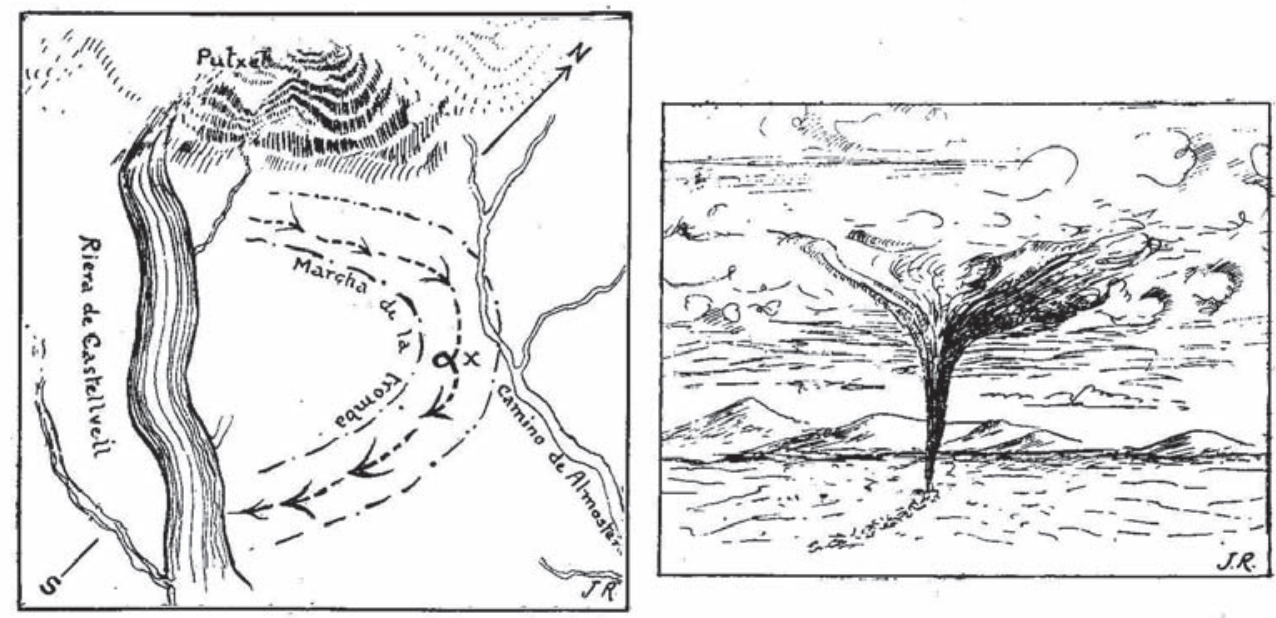

Marcha y aspecto de una tromba obseıvada por D. Juan Rovirosa, de Reus, en el término de Almoster (Tarragona), en 27 de junio de 1915.

FiguRa 3. Diagramas realizados por el observador Juan Rovirosa describiendo el tornado que se vivió en Almoster (Tarragona), el 27 de junio de 1915.

Fuente: BSAB, n. 52. 
estaciones, costeadas por la CIB, pero bajo la supervisión de la SAB: Ripoll, Ripoll-2, Ribes de Freser, Torelló, Manlleu, Roda de Ter, Montesquiu, Centelles, Anglès, Bonmartí, Fluvià, Castellfollit, Alguaire, Vallbona, Orís, Navàs, Sallent, Pont de Cabrianes, Pont de Vilomara y Viladecavalls-Calders (BSAB-59, 1916). Más tarde, en la localidad de Gelida se instala un nuevo pluviómetro. También la Sociedad General de Aguas de Barcelona, a partir de la primavera de 1916 proporciona datos pluviométricos de cinco estaciones cercanas a Barcelona (Barberà, Barcelona-Muntanya Pelada, Barcelona-Sant Andreu, Cornellà y Dosrius), mientras que el Sindicato General de Riegos del Canal de Urgell facilita datos de Mollerussa.

Durante este período de crecimiento de la red, además de la atención por las observaciones pluviométricas, también se impulsa el uso de nuevo instrumental, como es el caso de la veleta adoptada a finales de 1915 y de la cual aparece un detallado croquis en el boletín de diciembre de 1915 (BSAB-55, 1915). Igualmente, muchas estaciones inicialmente pluviométricas se transforman en termopluviométricas, como es el caso de la estación de Tivissa (en la comarca de la Ribera d'Ebre, Tarragona) a principios de 1913 y que dispone en la actualidad de más de 90 años de datos sin apenas lagunas. Las observaciones sin aparatos reciben también la atención de los observadores, y regularmente aparecen noticias sobre episodios de tiempo severo, como el paso de un tornado en Almoster (población cercana a Reus) el 27 de junio de 1915 y que describe con riqueza de detalles el observador Juan Rovirosa en el boletín número 52 (figura 3).

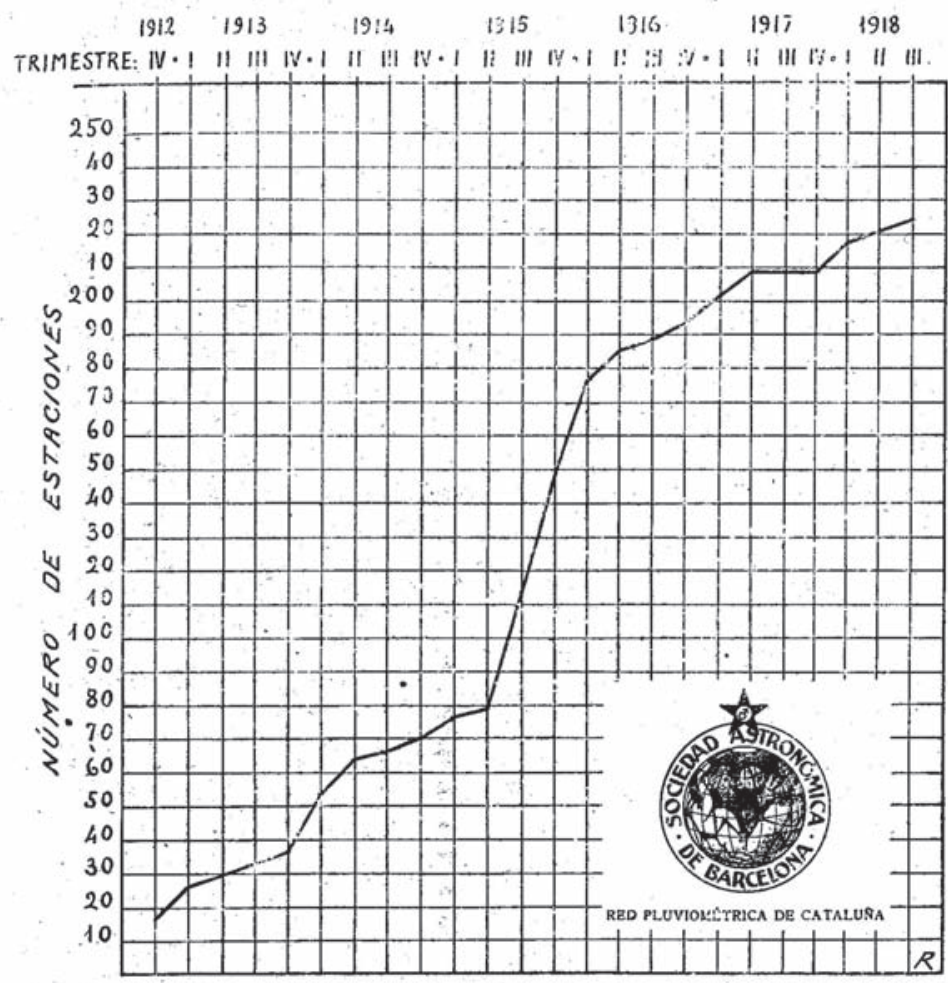

FIgURA 4. Evolución del número de estaciones integrantes de la Red Pluviométrica de Catalunya (BSAB-86, enero 1919). 
Como consecuencia de la extraordinaria labor de difusión de las observaciones pluviométricas en Catalunya, en tan sólo seis años se pasaron de 12 observaciones mensuales, en septiembre de 1912, a 224 en octubre de 1918 (figura 4). Lamentablemente, este espectacular crecimiento se vio bruscamente frenado a partir de 1918 debido a problemas económicos que harán inviable el sustento de la red.

\section{Crisis y disolución de la sociedad (1919-1923)}

A principios de 1919, la situación económica de la SAB va a cambiar de manera dramática. En la memoria presentada en enero de ese mismo año, el secretario Salvador Rauric, tras informar del crecimiento experimentado por la red pluviométrica desde 1912 manifiesta: «... es lamentable tener que consignar aquí, que el auxilio que durante dos ejercicios recibió de los Poderes centrales de la Sociedad Astronómica de Barcelona con destino a sostener y fomentar la Red pluviométrica de Cataluña, este año no nos ha sido concedido. De esta suerte la Sociedad Astronómica de Barcelona se encuentra enfrente del difícil problema de sostener exclusivamente, con sus limitados recursos ordinarios, esta vasta máquina de observación que, fiando quizá demasiado en el auxilio oficial, hemos desarrollado en Cataluña. Inútil creo hacer notar bien alto en este lugar, que esta situación compromete seriamente el sostenimiento y propagación de esta obra en Cataluña». (BSAB-86, enero 1919).

Muy pronto se harán realidad los malos presagios de Rauric, puesto que a los problemas económicos anunciados se le suman los derivados de los conflictos sociales que vive Barcelona, con continuas huelgas generales que afectan, en especial, a los talleres gráficos y provocan el aumento desorbitado del precio del papel. Por ello, a partir del último trimestre de 1918 y a lo largo de 1919, la Sociedad se ve obligada a reducir el espacio del boletín y se suspende temporalmente la publicación de las tablas mensuales de datos, conservándose la publicación de los mapas pluviométricos anuales. Incluso entre diciembre de 1919 y marzo de 1920, no se publica ni un solo boletín y es precisamente en este número de marzo (boletín número 93) en el que se publica el último mapa pluviométrico, el correspondiente al año 1918.

La crisis económica se manifiesta pronto en el crecimiento de la red que experimenta un estancamiento, manteniéndose inalterable el número de observatorios entre finales de 1918 y 1921. En 1921 se hace oficial, a través del último boletín publicado en octubre de 1921 (número 99), el traspaso de la Red Pluviométrica de Catalunya, gestionada por la SAB desde 1912, a la Mancomunitat de Catalunya y al Servei Meteorològic de Catalunya (SMC), que entró en funcionamiento de manera efectiva en marzo de ese mismo año. Los motivos aducidos eran sabidos: la incapacidad de continuar atendiendo a la conservación y fomento de esta obra colectiva por las dificultades económicas acuciantes. Así, el SMC recoge durante su primer año de vida el testimonio de gestionar una red de 224 observatorios, cohesionada y con un elevado grado de profesionalidad, a pesar del carácter amateur de sus integrantes. La Sociedad Astronómica de Barcelona continuó funcionando hasta 1923, año en que dejó de existir después de trece años de vida. Valga como homenaje final a su labor en la difusión de la observación meteorológica, la tabla 1 en la que se recogen todas aquellas estaciones pluviométricas creadas o fomentadas por la Sociedad, y los observadores a su cargo, entre 1910 y 1917. 
Tabla 1

ESTACIONES PLUVIOMÉTRICAS CREADAS O FOMENTADAS POR LA SAB ENTRE 1910 Y 1920, INDICANDO EL OBSERVADOR Y LA FECHA DE INICIO DE LAS OBSERVACIONES

\begin{tabular}{|c|c|c|c|}
\hline Observatorio & Comarca & Observador/es iniciales & Fecha inicio \\
\hline Agramunt & Urgell & Segimon Verdaguer & Ene, 1916 \\
\hline Agullana* & Alt Empordà & L. Marià Vidal & Oct, 1906 (Jul, 1912) \\
\hline Alp & Cerdanya & Ciprià Moner & Sep, 1915 \\
\hline Amposta & Montsià & Josep Via y Félix Via & Jul, 1911 \\
\hline Arenys de Mar & Maresme & Ricard Solà & Dic, 1915 \\
\hline Arenys de Munt & Maresme & Blai Llusà & Dic, 1915 \\
\hline Badalona & Barcelonès & $\begin{array}{l}\text { Joan Tapias; Teodor } \\
\text { Corominas }\end{array}$ & May, 1912 \\
\hline Barcelona - Gràcia & Barcelonès & Salvador Rauric & Oct, 1913 \\
\hline Barcelona - Vallvidrera & Barcelonès & Elies Barberà & Dic, 1915 \\
\hline Begur & Baix Empordà & Francesc Pi & Jul, 1911 \\
\hline Begur-Afores & Baix Empordà & Joaquim Batlle & Dic, 1912 \\
\hline Begur-Paradís (Fornells) & Baix Empordà & Bonaventura Sabater & Jun, 1916 \\
\hline Bell-lloc d'Urgell & Pla d'Urgell & Ramon Felip & Abr, 1916 (?) \\
\hline Benissanet & Ribera d'Ebre & Agustí Cot & Sep, 1911 \\
\hline Berga & Berguedà & Joaquim Serra & Mar, 1914 \\
\hline Bisbal, la & Baix Empordà & Joan B. Cuní & Abr, 1912 \\
\hline Blanes & Selva & Francesc Llac & Oct, 1911 \\
\hline Borges Blanques, les & Garrigues & Manuel Alemany & Dic, 1913 \\
\hline Cadaqués * & Alt Empordà & Sebastià Rahola & Gen, 1907 (Mar, 1913) \\
\hline Cambrils & Baix Camp & Carles Espinac & Ene, 1916 \\
\hline Cardona * & Bages & Antoni Mateu & Jun, 1908 (Jun, 1915) \\
\hline Castelló d'Empúries * & Alt Empordà & Fulgenci Vila & Nov, 1914 (Ene, 1916) \\
\hline Castelló de Rugat & Vall d'Albaida & Francesc Abines & Ene, 1913 \\
\hline Castellterçol & Bages & $\begin{array}{l}\text { Joan Sansano/Llorenç } \\
\text { Borràs }\end{array}$ & May, 1914 \\
\hline Cervera & Segarra & Frederic Vila & Nov, 1915 \\
\hline Ciutadilla & Urgell & Sr. Valls & (?) \\
\hline Collbató & Baix Llobregat & Pere Bacarisses & Ago, 1913 \\
\hline Corbera d'Ebre & Terra Alta & Pere Cortiella & Nov, 1912 \\
\hline
\end{tabular}




\begin{tabular}{|c|c|c|c|}
\hline Empúries (Sant Martí) & Alt Empordà & 1a Div. Hidrol. Forestal & Sep, 1913 \\
\hline Esparreguera & Baix Llobregat & Cristòfol Almirall & Gen, 1911 \\
\hline Espluga de Francolí, l' & Conca de Barberà & Blai Sans & Jun, 1915 \\
\hline Falset * & Priorat & $\begin{array}{l}\text { Josep Garreta/Josep } \mathrm{M}^{\mathrm{a}} \\
\text { Dalmau }\end{array}$ & Oct, 1896 (Dic, 1915) \\
\hline Far de Buda & Montsià & M. Díaz & Sep, 1916 \\
\hline Far St. Carles de Ràpita & Montsià & I. Llompart / M. Díaz & Ene, 1916 \\
\hline Garriga, la* & Vallès Oriental & D.J. Iglesias & Ene, 1908 (Sep, 1915) \\
\hline Garriga, la & Vallès Oriental & Frederic Wynn & Ene, 1916 \\
\hline Guissona * & Segarra & Pere Santaló & Abr, 1909 (Ene, 1916) \\
\hline Lleida & Segrià & Josep M $M^{a}$ Vicens & Feb, 1913 \\
\hline Llerona & Vallès Oriental & Pere Bou & Nov, 1915 (?) \\
\hline Llívia & Cerdanya & $\begin{array}{l}\text { Ricard Rius/Antoni } \\
\text { Esteva }\end{array}$ & Sep, 1915 \\
\hline Montblanc * & Conca de Barberà & Blai Sans/Josep Roselló & Sep, 1907 (Nov, 1915) \\
\hline Montgat & Maresme & Joan Guàrdia & Ene, 1912 \\
\hline Móra la Nova & Ribera d'Ebre & Rafael Borràs & Nov, 1915 \\
\hline Oliana * & Alt Urgell & Tomàs Espar & Mar, 1909 (Ene, 1916) \\
\hline Olot * & Garrotxa & Antoni Bolós & Ene, 1896 (Ene, 1914) \\
\hline Olost de Lluçanès & Osona & B. Darder/Dr. Moré & Nov, 1915 \\
\hline Os de Balaguer* & Noguera & Hermengildo Cirera & Ago, 1907 (Sep, 1915) \\
\hline Palafrugell * & Baix Empordà & Ramir Medir & Ene, 1897 (Mar, 1912) \\
\hline Palamós & Baix Empordà & Zoilo Costart & Jul, 1911 \\
\hline Palautordera & Vallès Oriental & Benet Adroer & Feb, 1914 \\
\hline Palma de Mallorca & Illa de Mallorca & Gregori Guasp & Ago, 1914 \\
\hline Pasteral, el * & Selva & Joaquim Solé & Ene, 1902 (Mar, 1912) \\
\hline Pobla de Lillet & Berguedà & Conrad Prat & Mai, 1914 \\
\hline Pont de Suert* & Alta Ribagorça & Carles Saura & Jul, 1907 (Abr, 1916) \\
\hline Porrera * & Priorat & Jaume Monlleó & Oct, 1907 (Nov, 1917) \\
\hline Puigcerdà * & Cerdanya & Armengol Ferrer & Dic, 1895 (Sep, 1915) \\
\hline Prats i Sansor & Cerdanya & Pere Pons & Dic, 1913 \\
\hline Ripoll-Llaés & Ripollès & Joan Babot & Dic, 1913 \\
\hline Sabadell $*$ & Vallès Occidental & Escoles Pies & Oct, 1896 (Nov, 1915) \\
\hline Sant Feliu de Guíxols & Baix Empordà & FFCC S. Feliu a Girona & Feb, 1914 \\
\hline
\end{tabular}




\begin{tabular}{|c|c|c|c|}
\hline Sant Feliu de Llobregat* & Baix Llobregat & $\begin{array}{l}\text { Juan Planas/Manuel } \\
\text { Tamayo }\end{array}$ & Ene, 1906 (Ene, 1912) \\
\hline Sant Feliu de Pallerols * & Garrotxa & M. Bosch Mir & Ene, 1907 (Ene, 1916) \\
\hline Sant Hilari Sacalm* & Selva & Fernando Cid & Ene, 1907 (Nov, 1912) \\
\hline Sant Pere de Ribes & Garraf & Josep Puig Miret & Nov, 1915 \\
\hline Sant Quintí de Mediona & Alt Penedès & Alexandre Fortuny & Dic, 1915 \\
\hline Santa Fe del Montseny & Vallès Oriental & Teresa Martí & Ene, 1916 \\
\hline Santuari del Miracle & Solsonès & Àngel Salut & Ene, 1916 \\
\hline Santuari dels Àngels & Gironès & Joaquim Clotas & Ene, 1916 \\
\hline Solsona & Solsonès & Narcís Ballescà & Dic, 1915 \\
\hline Tàrrega & Urgell & J. Torregrossa/J. Flaquer & Jun, 1914 \\
\hline St. Andreu Llavaneres & Maresme & August Folch & Nov, 1912 \\
\hline Teià * & Maresme & Jacint Barrera & Ene, $1900($ Ene, 1911) \\
\hline Tivissa & Baix Ebre & Rossend Loran & Oct, 1911 \\
\hline Torroella de Montgrí & Baix Empordà & Pere Blasi & Mar, 1912 \\
\hline Vallfogona de Riucorb & Conca de Barberà & Josep J. Ursul & Dic, 1913 \\
\hline Valls * & Alt Camp & Germans de Sant Gabriel & Sep, 1907 (Jul, 1915) \\
\hline Vandellós & Baix Camp & $\begin{array}{l}\text { Josep Ballvé i Josep } \\
\text { Clapés }\end{array}$ & May, 1912 \\
\hline Vendrell, el & Baix Penedès & Jaume Palau Badia & Sep, 1915 \\
\hline Vic & Osona & Josep Pratdesaba & Nov, 1914 \\
\hline Vielha * & Val d'Aran & $\begin{array}{l}\text { Germans Escoles } \\
\text { Cristianes }\end{array}$ & Jun, 1907 (Sep, 1915) \\
\hline Viladrau & Osona & Antoni Ariet & Ene, 1914 \\
\hline Vilert-Banyoles * & Pla de l'Estany & Josep Anglada & Ene, 1867 (Dic, 1912) \\
\hline Villena & Alt Vinalopó & Tomàs Giner & Nov, 1911 \\
\hline
\end{tabular}

* Estaciones de la red pluviométrica de Patxot, que a partir de la fecha que se indica entre paréntesis funcionan con material (pluviómetros) costeado por la SAB u observadores coordinados por la SAB.

(?) No se sabe con certeza la fecha inicial.

Fuente: elaboración propia a partir de los boletines de la SAB.

\section{EI bagaje de la Sociedad}

La perspectiva de la historia permite valorar justamente la labor y contribución de las personas e instituciones en el desarrollo de la ciencia. En este sentido, la Sociedad Astronómica de Barcelona dejó para las décadas posteriores un bagaje de incalculable valor. En 
primer lugar, supo recoger y aunar los esfuerzos de personas y asociaciones precedentes tales como la red de Rafael Patxot o la Granja Experimental de Barcelona. Supo coordinar y ampliar una red de observatorios que perduró casi intacta durante más de un cuarto de siglo y supo despertar el interés del capital privado para dar impulso a esta empresa. La $\mathrm{SAB}$ actuó como un verdadero servicio meteorológico, dictando instrucciones para las observaciones y planificando la ubicación de los puntos de observación con criterios científicos. Pero además de su bagaje científico cabe destacar su vocación divulgadora y los esfuerzos por hacer llegar la trascendencia de los estudios climáticos a la ciudadanía. Actuó como catalizador de la demanda social creciente por el conocimiento del medio natural del país, tal y como lo fueron también las agrupaciones excursionistas que nacieron en esa época. Pero sobre todo, gracias a la gestión de sus colaboradores y socios, y a pesar de las limitaciones económicas, ha sido posible que lleguen hasta nuestros días un buen número de datos climáticos de extraordinario valor científico y patrimonial. Su explotación merece toda nuestra atención.

\section{Agradecimientos}

Al Dr. Mariano Barriendos por sus útiles comentarios durante la confección del presente artículo.

\section{Bibliografía}

BARRIENDOS, M. (2001): «Francesc Salvà i Campillo (1751-1828). En record a la seva figura i tarannà científic en el 250è aniversari del seu naixement», en ACAM: VII Jornades de Meteorologia, Vilafranca del Penedès, pp.13-22 (en catalán).

BARRIENDOS, M.; GÓMEZ, B. y PEÑA, J.C. (1997): «Old series of meteorological readings for Madrid and Barcelona (1780-1860). Documentary and observed characteristics», en MARTÍNVIDE, J. (ed.) Advances in Historical Climatology in Spain, Oikos-Tau, Barcelona, pp. 157-172. Traducción en castellano en la misma obra, pp. 47-62.

BOLETÍN DE LA SOCIEDAD ASTRONÓMICA DE BARCELONA, BSAB (1910-1921), Números de 1 a 99 . Barcelona.

FEBRER, J. (1930): Atlas pluviomètric de Catalunya. Memòries Patxot, vol. 1. Institució Patxot. Barcelona, $518 \mathrm{pp}$.

FONTSERÈ, E. (1911): Boletín de la Sociedad Astronómica de Barcelona, n. , pp.

GARCÍA DE PEDRAZA, L. y GIMÉNEZ DE LA CUADRA, J.M. (1985): Notas para la historia de la Meteorología en España. Instituto Nacional de Meteorología. Secretaría General, Madrid, $144 \mathrm{pp}$.

JARDÍ, R. (1912): Observaciones del eclipse solar del 17 de abril de 1912. Boletín de la Sociedad Astronómica de Barcelona, n. 20, pp. 512-518.

PATXOT, R. (1908): Meteorologia Catalana. Observacions de Sant Feliu de Guíxols. Resultats de 1896 (parcial) al 1905. Barcelona, L’Avenç, 307 pp.

PATXOT, R. (1912): Pluviometria Catalana. Resultats del cinqueni 1906-1910 publicats amb la col-laboració dels observadors de Catalunya per Rafael Patxot i Jubert. Sant Feliu de Guíxols - Observatori Català., 311 pp.

PUIG, C. (1995): Llorenç Presas i Puig (1811-1875). La matemàtica aplicada en CAMARASA, J.M., ROCA, A. (dir.) Ciència i Tècnica als Països Catalans. Una aproximació bibliogràfica als darrers 150 anys. Barcelona, Fundació Catalana per la Recerca, pp. 145-180 (en catalán).

ROCA, A.; BATLLÓ, J. y ARÚS, J. (2004): «Eduard Fontserè i Riba. Promotor de la meteorologia professional Catalana» en ACAM: X Jornades de Meteorologia Eduard Fontserè, Barcelona, pp. 11-63 (en catalán). 
RODRÍGUEZ, R.; BARRIENDOS, M.; JONES, P.D.; MARTÍN-VIDE, J. y PEÑA, J.C. (2001): «Long pressure series for Barcelona (Spain). Daily reconstruction and monthly homogenization», International Journal of Climatology, vol. 21, núm. 13, pp. 1693-1704.

SUREDA, V. (2003): «Hermenegildo Gorría: la seva obra en relació amb la meteorologia catalana» en ACAM: IX Jornades de Meteorologia Eduard Fontserè, Barcelona, pp. 19-24 (en catalán). 